\title{
SINGULAR VALUE SELECTION AND GENERALIZED CROSS VALIDATION IN MULTI-FREQUENCY SEISMIC DIFFRACTION TOMOGRAPHY FOR $\mathrm{CO}_{2}$ INJECTION MONITORING
}

\author{
Caio Jean Matto Grosso da Silva ${ }^{1}$ and Amin Bassrei
}

\begin{abstract}
Regardless of whether the cause of the greenhouse effect is anthropogenic, carbon dioxide $\left(\mathrm{CO}_{2}\right)$ exacerbates global warming because it contributes directly to the increased temperature of the planet. In a geologic context, $\mathrm{CO}_{2}$ can occur in conjunction with porous oil reservoirs. Thus, what should be done with $\mathrm{CO}_{2}$ ? Two techniques have shown great potential. The first is carbon capture and storage (CCS), which involves injecting gas into saline aquifers, depleted reservoirs and coal seams. The second technique is enhanced oil recovery (EOR), which involves injecting $\mathrm{CO}_{2}$ into heavy oil reservoirs to reduce the oil's viscosity and to increase the amount recovered. Once initiated, the injection of carbon dioxide requires periodic monitoring. In this paper, we propose the use of well-to-well diffraction tomography with a multi-frequency approach for mapping the velocity changes associated with $\mathrm{CO}_{2}$ injection in a reservoir. Diffraction tomography is considered to be an ill-posed inverse problem. To avoid this situation, we separately applied two techniques. The first uses an optimal number of singular values and discards the smallest singular values to increase the stability of the inverse problem. The second method is based on regularization by derivative matrices, and we used generalized cross validation to select the optimal parameter. The results obtained for the multi-frequency approaches have proven reliable based on the applied synthetic models. Moreover, the selection of singular values and regularization by matrices methods were demonstrated to be valid for seismic diffraction tomography.
\end{abstract}

Keywords: seismic diffraction tomography, reservoir monitoring, Gassmann's equation, $\mathrm{CO}_{2}$ injection.

RESUMO. Independentemente se a causa do efeito de estufa é antropogênico, o dióxido de carbono $\left(\mathrm{CO}_{2}\right)$ agrava 0 aquecimento global porque contribui diretamente para 0 aumento da temperatura do planeta. Em um contexto geológico, $0 \mathrm{CO}_{2}$ pode ocorrer em conjunto com reservatórios de petróleo porosos. Assim, 0 que deve ser feito com $\mathrm{CO}_{2}$ ? Duas técnicas têm mostrado grande potencial. A primeira é a captura e armazenamento de carbono (CCS), que envolve a injeção de gás em aquíferos salinos, reservatórios esgotados e camadas de carvão. A segunda técnica é a recuperação avançada de petróleo (EOR), que envolve a injeção de $\mathrm{CO}_{2}$ em reservatórios de óleo pesado para reduzir a viscosidade do óleo e para aumentar a quantidade recuperada. Uma vez iniciada, a injeção de dióxido de carbono exige um acompanhamento periódico. Neste trabalho, propomos o uso da tomografia de difração, na geometria de aquisição poço a poço, com uma abordagem multifrequência para mapear as mudanças de velocidade associadas à injeção de $\mathrm{CO}_{2}$ em um reservatório. A tomografia de difração é considerada como um problema inverso mal posto. Para atenuar essa situação, duas técnicas foram aplicadas separadamente. A primeira usa um número ótimo de valores singulares e descarta os menores valores singulares para aumentar a estabilidade do problema inverso. A segunda técnica é baseada na regularização por matrizes de derivadas, onde foi utilizada a validação cruzada generalizada para selecionar o parâmetro ótimo de regularização. Os resultados obtidos para a abordagem multifrequência apresentam-se confiáveis com base nos modelos sintéticos estudados. Além disso, a seleção de valores singulares e a regularização por matrizes de derivadas mostraram-se válidas em tomografia sísmica de difração.

Palavras-chave: tomografia sísmica de difração, monitoramento de reservatórios, equação de Gassmann, injeção de $\mathrm{CO}_{2}$.

\footnotetext{
1PETROBRAS, Avenida Elias Agostinho, 665, Imbetiba, 27913-350 Macaé, RJ, Brazil. Phone: +55(22) 3377-2937 - E-mail: cjmgs@petrobras.com.br

${ }^{2}$ Universidade Federal da Bahia, Research Center in Geophysics and Geology \& Institute of Geosciences and National Institute of Science and Technology in Petroleum Geophysics, Rua Barão de Jeremoabo, s/n, Ondina, 40170-115 Salvador, BA, Brazil. Phone: +55(71) 3283-8508 - E-mail: bassrei@ufba.br
} 


\section{INTRODUCTION}

The injection of $\mathrm{CO}_{2}$ into porous reservoirs is a recent technique that reduces the emission of this gas into the atmosphere. Two types of $\mathrm{CO}_{2}$ injection exist: carbon capture and storage (CCS), which consists of $\mathrm{CO}_{2}$ storage in saline aquifers, and enhanced oil recovery (EOR), which involves gas injection to increase the recovery rate in reservoirs with heavy oil. In this context, diffraction tomography can estimate variations in the velocity field. We intend to validate its use as an aid in the monitoring of injection fluid in a reservoir.

Diffraction tomography is an inversion technique that allows the estimation of the velocity distribution in the subsurface. The input data are the amplitudes of seismic signals recorded in the receivers. Diffraction tomography is a nonlinear problem that can be solved, for example, by the Born approximation, although this is only valid when there is little variation in the speed of the medium. In addition, diffraction tomography is an ill-posed inverse problem, requiring the use of certain types of adjustment or regularization to reduce the ill-conditioned nature of the problem. Moreover, for the study of substitution fluids within a reservoir, we must use Gassmann's equation, which is an important tool for estimating velocity changes in a reservoir due to the substitution of a given fluid for another. The image, resulted from the subtraction between two stages, shows the $\mathrm{CO}_{2}$ migration, that is, to what physical extent and to what saturation level the $\mathrm{CO}_{2}$ front is evolving within the layer or layers of interest. This procedure categorizes geophysical tomography as a reservoir monitoring tool, which is, in general more precise quantitatively and with greater resolution than the seismic tomography results in a reservoir characterization application.

A previous work (Santos et al., 2009) used single-frequency diffraction tomography with trigonal meshes to monitor $\mathrm{CO}_{2}$ leakage, whereas we also desired to estimate the approximate shape of the layer containing the $\mathrm{CO}_{2}$. In this work, we used a multi-frequency approach with rectangular blocks without a priori assumptions, and the velocity change is calculated via the variation in petrophysical parameters. We chose a selection of singular values to improve the ill-conditioned nature of the inverse matrix. The optimum number of singular values was determined by energy and entropy criteria with respect to the estimated parameters in the model. The RMS error between the true and estimated model parameters was computed to validate these criteria for energy and entropy. The second approach to adjust or regularize the ill-posed inverse problem involved regularization via derivative matrices (Twomey, 1963; Tikhonov \& Arsenin, 1977). We employed generalized cross validation (GCV) to determine the optimum regularization parameter $\lambda$. Other methodologies can be used to determine $\lambda$ such as the L-curve and the $\Theta$-curve (Santos \& Bassrei, 2007).

The objective of this study is to validate the application of well-to-well diffraction tomography to monitor the injection of $\mathrm{CO}_{2}$ in sandstone reservoirs and to obtain more representative results for saturation values, even if the results are qualitative. Therefore, numerical simulations were performed with data contaminated with noise.

This article is divided as follows: First, the theory of inversion and related problems are presented, and the selection of singular values and regularization by derivative matrices are combined in the GCV method to choose the regularization parameter. Second, Gassmann's equation is introduced to address the effects of the substitution of fluids in a reservoir. Then, the theory of diffraction tomography and its limitations due to the use of the Born approximation are also introduced. A matrix formulation and the multi-frequency approach to diffraction tomography are presented. Then, the results of the numerical simulations, involving four stages of $\mathrm{CO}_{2}$ saturation and water within the reservoir, are presented and discussed.

\section{Inverse Problems}

Inverse problems are generally ill-posed; that is, either a solution does not exist or, if a solution does exist, then it is not unique or is not stable (or both). In the analysis of physical phenomena, we can classify the relationship between data parameters and model parameters into two classes: (i) direct modeling, where the data are predicted from a given model, and (ii) inverse modeling, where the model parameters are determined from observed data.

Inverse problems, either linear, piecewise linear or linearized, can be formulated as a linear system of equations (Menke, 1989):

$$
\mathbf{d}=\mathbf{A m}
$$

where $\mathbf{d}$ is the data parameter vector, $\mathbf{m}$ is the model parameter vector and $A$ is a $M \times N$ matrix that relates the $M$ data parameters to the $N$ model parameters.

Assuming that the matrix $\mathbf{A}$ is known and invertible, we can find the solution as follows:

$$
\mathbf{m}=\mathbf{A}^{-1} \mathbf{d} .
$$

The matrix $\mathbf{A}^{-1}$ will exist if the matrix $\mathbf{A}$ is square and has full rank. Because this situation is a very rare in geophysics, the initial problem must be reformulated to obtain a feasible solution. 


\section{Singular Value Decomposition and Singular Value Selection}

Singular value decomposition (SVD) is a tool for estimating the inverse of a matrix that is not square and/or does not have full rank. The SVD technique comprises a decomposition of a given matrix $A$ in three other matrices, which are given by the following:

$$
\mathbf{A}=\mathbf{U} \boldsymbol{\Sigma} \mathbf{V}^{\mathbf{T}},
$$

where $\boldsymbol{\Sigma}$ is a diagonal matrix having the singular values of matrix $\mathbf{A}$ arranged in descending order and the matrices $\mathbf{U}$ and $\mathbf{V}$ are orthonormal matrices. Thus, from the properties of the orthonormal matrices and $\boldsymbol{\Sigma}$, we can determine a generalized inverse matrix (Penrose, 1955), as follows:

$$
\mathbf{A}^{+}=\mathbf{V} \boldsymbol{\Sigma}^{+} \mathbf{U}^{\mathbf{T}},
$$

where $\boldsymbol{\Sigma}^{+}$is a diagonal matrix containing the reciprocals of the singular values of the matrix $\mathbf{A}$.

The stability of a matrix can be measured by the ratio between its largest and its smallest singular values. Clearly, very small singular values will lead to greater instability in the inverse problem. In this work, the smallest singular values, which compromise the quality of the solution, are removed.

The energy of the model shows the stability of a particular solution (Silva and Bassrei, 2009) and can be written as follows:

$$
E=\sum_{i=1}^{N}\left(m_{i}^{e s t}\right)^{2},
$$

where $m_{i}^{\text {est }}$ is the $i$-th estimated model parameter and $N$ is the number of model parameters.

The concept of entropy was developed by the German Rudolf Clausius, in the context of classical thermodynamics, and later the Austrian physicist Ludwig Boltzmann gave the statistical interpretation of entropy. It can be defined in several forms. According to the increase in entropy, the universe and all the closed systems, tend naturally to deteriorate and lose the clearness, to change from a state of minimum probability to another of maximum probability, from a state of organization and differentiation, in which exist forms and distinctions, to a state of chaos. In the contingent universe, the order is less probable. The role of entropy is in such a way that Jaynes (1957), considers it a primitive physical concept, even more fundamental than the concept of energy. We make use of entropy in the framework of information theory (IT) as defined by Shannon in 1948 (Shannon \& Weaver, 1949), and given by the following equation:

$$
H=\sum_{i=1}^{N} m_{i}^{e s t} \log \left(\frac{1}{m_{i}^{e s t}}\right),
$$

where, again, $m_{i}^{e s t}$ is the $i$-th estimated model parameter.

It is interesting to find the distribution which maximizes the entropy. Because entropy is a measure of uncertainty, the probability distribution which generates maximum uncertainty will have maximum entropy. In the absence of prior information, Jaynes (1957), stated that the maximum entropy is the less biased estimate from a given information.

The RMS error between the true and estimated model parameters is given by the following equation:

$$
E_{m}=\frac{1}{N} \sqrt{\sum_{i=1}^{N}\left(m_{i}^{\text {true }}-m_{i}^{\text {est }}\right)^{2}}
$$

where $m_{i}^{\text {true }}$ is the $i$-th true model parameter and $m_{i}^{e s t}$ is the estimated model parameter. The $E_{m}$ estimator will be used for quality evaluation of the inversion results. It will not be used for the choice of the optimal number of singular values because the parameter $m_{i}^{\text {true }}$ is unknown in real situations.

\section{Regularization by Derivative Matrices and Generalized Cross Validation}

One way to improve the stability of the solution is minimally limiting the variation in model parameters. One can use the difference between physically adjacent model parameters as an approximation of the first derivative. The sum of these values can be defined as the flatness $\mathbf{l}_{\mathbf{1}}$ of the solution:

$$
\begin{aligned}
\mathbf{l}_{\mathbf{1}} & =\left(\begin{array}{ccccccc}
-1 & 1 & 0 & \cdots & 0 & 0 & 0 \\
0 & -1 & 1 & \cdots & 0 & 0 & 0 \\
\vdots & \vdots & \vdots & \vdots & \ddots & \vdots & \vdots \\
0 & 0 & 0 & 0 & 0 & -1 & 1
\end{array}\right)\left(\begin{array}{c}
m_{1} \\
m_{2} \\
\vdots \\
m_{N}
\end{array}\right) \\
& =\mathbf{D}_{\mathbf{1}} \mathbf{m} .
\end{aligned}
$$

Alternatively, one can choose to use the roughness $\mathbf{l}_{\mathbf{2}}$ of the model parameters by using an approximation of the second derivative matrix $\mathbf{D}_{\mathbf{2}}$ :

$$
\begin{aligned}
\mathbf{l}_{\mathbf{2}} & =\left(\begin{array}{ccccccccc}
1 & -2 & 1 & 0 & \cdots & 0 & 0 & 0 & 0 \\
0 & 1 & -2 & 1 & \cdots & 0 & 0 & 0 & 0 \\
\vdots & \vdots & \vdots & \vdots & \ddots & \vdots & \vdots & \vdots & \vdots \\
0 & 0 & 0 & 0 & \cdots & 0 & 1 & -2 & 1
\end{array}\right)\left(\begin{array}{c}
m_{1} \\
m_{2} \\
\vdots \\
m_{N}
\end{array}\right) \\
& =\mathbf{D}_{\mathbf{2}} \mathbf{m} .
\end{aligned}
$$

The value of $L_{n}$, either flatness $(n=1)$ or roughness $(n=2)$, related to the model parameter is as follows:

$$
L_{n}=\left\|\mathbf{l}_{\mathbf{n}}\right\|_{2}^{2}=\left(\mathbf{D}_{\mathbf{n}} \mathbf{m}\right)^{T}\left(\mathbf{D}_{\mathbf{n}} \mathbf{m}\right),
$$


where $n$ is the order of the derivative matrix. One can then define an objective function $\Phi(m)$ (Bassrei \& Rodi, 1993) as follows:

$$
\Phi(\mathbf{m})=\mathbf{e}^{\mathbf{T}} \mathbf{e} .
$$

Using the relation $\mathbf{e}=\mathbf{d}-\mathbf{A m}$ and substituting equation (10) into equation (11), we obtain the following:

$$
\begin{gathered}
\Phi(\mathbf{m})= \\
(\mathbf{d}-\mathbf{A m})^{T}(\mathbf{d}-\mathbf{A} \mathbf{m})+\lambda\left(\mathbf{D}_{\mathbf{n}} \mathbf{m}\right)^{T}\left(\mathbf{D}_{\mathbf{n}} \mathbf{m}\right),
\end{gathered}
$$

where $\lambda$ is a positive constant known as the regularization parameter, which represents the regularization intensity that is applied to provide a satisfactory solution. When $\lambda=0$, equation (12) is the least squares method. If $\lambda \neq 0$ and the regularization order is zero, i.e., $L_{0}=\left\|\mathbf{l}_{\mathbf{0}}\right\|_{2}^{2}=\left(\mathbf{D}_{\mathbf{0}} \mathbf{m}\right)^{T}\left(\mathbf{D}_{\mathbf{0}} \mathbf{m}\right)$, the equation becomes the so-called damped least squares method.

Minimizing the objective function shown in equation (12), we obtain the following:

$$
\begin{gathered}
\frac{\partial \Phi(\mathbf{m})}{\partial \mathbf{m}}= \\
2 \mathbf{A}^{\mathbf{T}} \mathbf{A m}^{\text {est }}-2 \mathbf{A}^{\mathbf{T}} \mathbf{d}+2 \lambda \mathbf{D}_{\mathbf{n}}^{\mathbf{T}} \mathbf{D}_{\mathbf{n}} \mathbf{m}^{\text {est }} .
\end{gathered}
$$

Setting this equation equal to zero, we can find the value of $\mathbf{m}^{\text {est }}$ that produces the smallest error:

$$
\mathbf{m}^{e s t}=\left(\mathbf{A}^{\mathbf{T}} \mathbf{A}+\lambda \mathbf{D}_{\mathbf{n}}^{\mathbf{T}} \mathbf{D}_{\mathbf{n}}\right)^{-1} \mathbf{A}^{\mathbf{T}} \mathbf{d} .
$$

The generalized cross validation method was proposed by Craven \& Wahba (1979) as a tool for choosing the optimal value of parameter $\lambda$. The GCV estimate comes from ordinary cross validation (OCV), which is based on the concept of leaving-oneout, i.e., removing an element of the data vector and calculating the regularized solution that minimizes the objective function. The OCV estimate for $\lambda$ is defined as the value that minimizes the following function:

$$
V_{0}(\lambda)=\frac{1}{M} \sum_{k=1}^{M}\left[d_{k}^{o b s}-d_{k}\left(\mathbf{m}_{\lambda}^{k}\right)\right]^{2},
$$

where the index $k$ corresponds to the $k$-th element that was removed from the data vector. If the value of $\lambda$ is optimal, then the $k$-th element of the solution (model parameter vector) will predict the failure; that is, $\mathbf{m}_{\lambda}^{k}$ is the solution that minimizes the error. Considering the linear relationship, the influence matrix is defined as follows (Craven \& Wahba, 1979):

$$
\mathbf{A m}_{\lambda}=\mathbf{B}(\lambda) \mathbf{d} .
$$

One way to evaluate $V_{0}(\lambda)$ more efficiently, without the need to solve the inverse problem for each observation value, is presented by Wahba (1990) in the form of the following equation:

$$
\sum_{k=1}^{M}\left[d_{k}^{o b s}-d_{k}\left(\mathbf{m}_{\lambda}^{k}\right)\right]=\sum_{k=1}^{M} \frac{d_{k}^{o b s}-d_{k}\left(\mathbf{m}_{\lambda}\right)}{1-b_{k k}(\lambda)},
$$

where

$$
b_{j j}(\lambda)=\frac{d_{k}(\mathbf{m})-d_{k}\left(\mathbf{m}_{\lambda}^{k}\right)}{d_{j}^{o b s}-d_{k}\left(\mathbf{m}_{\lambda}^{k}\right)},
$$

$b_{j j}$ being the $j$-th element of the main diagonal of the influence matrix $\mathbf{B}(\lambda)$. Therefore,

$$
V_{0}(\lambda)=\frac{1}{M} \sum_{k=1}^{M} \frac{\left[d_{k}^{o b s}-d_{k}\left(\mathbf{m}_{\lambda}\right)\right]^{2}}{\left[1-b_{k k}(\lambda)\right]^{2}} .
$$

The GCV function is obtained based on the following relationship: $\mu_{1}(\lambda)=1 / M \sum_{i=1}^{M} b_{i i}(\lambda)=(1 / M) \operatorname{Tr}[\mathbf{B}(\lambda)]$. Thus,

$$
\begin{aligned}
V(\lambda) & =\frac{1}{M} \sum_{k=1}^{M} \frac{\left[d_{k}^{o b s}-d_{k}\left(\mathbf{m}_{\lambda}\right)\right]^{2}}{\left[1-\mu_{1}(\lambda)\right]^{2}} \\
& =\frac{\left\|\mathbf{d}^{o b s}-\mathbf{d}\left(\mathbf{m}_{\lambda}\right)\right\|}{\left[\frac{1}{M} \operatorname{Tr}(\mathbf{I}-\mathbf{B}(\lambda)]^{2}\right.},
\end{aligned}
$$

if

$$
\mathbf{m}_{\lambda}=\left(\mathbf{A}^{\mathbf{T}} \mathbf{A}+\lambda \mathbf{D}_{\mathbf{n}}^{\mathbf{T}} \mathbf{D}_{\mathbf{n}}\right)^{-1} \mathbf{A}^{\mathbf{T}} \mathbf{d}^{o b s},
$$

where the matrix $\mathbf{B}(\lambda)$ is defined as

$$
\mathbf{B}(\lambda)=\mathbf{A}\left(\mathbf{A}^{\mathbf{T}} \mathbf{A}+\lambda \mathbf{D}_{\mathbf{n}}^{\mathbf{T}} \mathbf{D}_{\mathbf{n}}\right)^{-1} \mathbf{A}^{\mathbf{T}} .
$$

\section{Seismic Diffraction Tomography}

The propagation of a certain scalar perturbation $\Psi(\mathbf{r}, t)$ through a medium with constant density and variable velocity given by $c(\mathbf{r})$ can be modeled by the wave equation (Devaney, 1984; Harris, 1987; Wu \& Toksöz, 1987):

$$
\nabla^{2} \Psi(\mathbf{r}, t)=\frac{1}{c^{2}(\mathbf{r})} \frac{\partial^{2} \Psi(\mathbf{r}, t)}{\partial t^{2}},
$$

where $\mathbf{r}$ is the position vector within the considered model, $t$ represents time and $\nabla^{2}$ is the Laplacian operator. We can use harmonic waves in time represented by exponentials to find a soIution to the wave equation (Lo \& Inderwiesen, 1994):

$$
\Psi(\mathbf{r}, t)=e^{-j \omega t} P(\mathbf{r}, t),
$$

where $P(\mathbf{r}, t)$ is the wave amplitude defined as a function of angular frequency. 
Substituting equation (24) into equation (23), we have the Helmholtz equation, which describes the wave scattering and is given by the following:

$$
\nabla^{2} P(r, \omega)+\kappa^{2}(r, \omega) P(r, \omega)=0 .
$$

This equation is also found by the application of Fourier transform on the wave equation in the time domain. The parameter $\kappa(\mathbf{r}, \omega)$ is the magnitude of the wavenumber at position $\mathbf{r}$ and is defined by the following:

$$
\kappa(\mathbf{r}, \omega)=\frac{\omega}{c(\mathbf{r})} .
$$

Note that the wavenumber and wave amplitude in equation (25) depend on the value of the angular frequency $\omega$. However, to simplify the notation, we will write $P(\mathbf{r}, \omega)$ and $\kappa(\mathbf{r}, \omega)$ as $P(\mathbf{r})$ and $\kappa(\mathbf{r})$, respectively, but keep in mind that there is a dependence on the angular frequency $\omega$.

The Lippmann-Schwinger equation is a nonlinear equation which relates the data function $P_{S}(\mathbf{r})$ to the model function $M(\mathbf{r})$. The former function is called the scattered wavefield. The scattering problem consists of an incident wave, $P_{I}(\mathbf{r})$, that propagates from the source within a medium with a constant background velocity, given by $c_{0}$. The objective is to image the 2-D medium $A$ in which the velocity contrast is $c(\mathbf{r})$. Any portion of the medium where $c(\mathbf{r}) \neq c_{0}$ will act as a secondary source and will scatter the incident wavefield. The scattered wavefield travels from the heterogeneity to the receivers, where it is registered. Other approaches can also be used in situations where the background velocity is not constant, including methods such as those of Dickens (1994) and Harris \& Wang (1996), who considered a layered medium.

The wavefield recorded by a receiver is the total field $P_{T}(\mathbf{r})$, which is the sum of the incident and the scattered wavefields:

$$
P_{T}(\mathbf{r})=P_{I}(\mathbf{r})+P_{S}(\mathbf{r}) .
$$

For a constant density model, equation (25) describes the propagation of the total wavefield:

$$
\left[\nabla^{2}+\kappa^{2}(\mathbf{r})\right] P_{T}(\mathbf{r})=0 .
$$

The wavenumber associated with the medium with constant background velocity is

$$
\kappa_{0}=\frac{\omega}{c_{0}}
$$

Thus, we can define $\kappa^{2}(\mathbf{r})$ as

$$
\kappa^{2}(\mathbf{r})=\kappa_{0}^{2}+\kappa_{0}^{2} M(\mathbf{r}),
$$

where

$$
M(\mathbf{r})=\left[\frac{c_{0}^{2}}{c^{2}(\mathbf{r})}-1\right] .
$$

The model function $M(\mathbf{r})$ in equation (31) defines a deviation from the constant wavenumber $\kappa_{0}^{2}$; that is, if $c(\mathbf{r})=c_{0}$ in equation (31), then $M(\mathbf{r})=0$, and there is no scattering. From equations (27), (28) and (30), we obtain the Helmholtz equation for $P_{S}(\mathbf{r})$ :

$$
\left[\nabla^{2}+\kappa^{2}(\mathbf{r})\right] P_{S}(\mathbf{r})=\kappa_{0}^{2} M(\mathbf{r})\left[P_{I}(\mathbf{r})+P_{S}(\mathbf{r})\right] .
$$

Solving equation (32) directly for $P_{S}(\mathbf{r})$ is a complicated task. We can find an approximation to the solution using the properties of Green's function (Lo \& Inderwiesen, 1994). If $G(r)$ is Green's function, i.e., the field registered at the observation point $r$ and caused by a unitary delta function source at $\mathbf{r}^{\prime}$, then the field at $\mathbf{r}$ caused by a distribution of delta function sources is the integral of $G(\mathbf{r})$ over the entire region occupied by the source. The solution of equation (32) at $\mathbf{r}$ for a unitary source generated in $\mathbf{r}^{\prime}$ with wavenumber $\kappa_{0}$ must first be calculated:

$$
\left[\nabla^{2}+\kappa^{2}(\mathbf{r})\right] G\left(\mathbf{r} \mid \mathbf{r}^{\prime}\right)=-\delta\left(\mathbf{r}-\mathbf{r}^{\prime}\right) .
$$

Green's function $G\left(\mathbf{r} \mid \mathbf{r}^{\prime}\right)$ represents the solution at the position $\mathbf{r}$ for an impulse at $\mathbf{r}^{\prime}$, which corresponds to the scatter point (Lo \& Inderwiesen, 1994). The solution of equation (33) for a two-dimensional domain is given by the following equation:

$$
G\left(\mathbf{r} \mid \mathbf{r}^{\prime}\right)=\frac{j}{4} H_{0}^{(1)}\left(\kappa_{0}\left|\mathbf{r}-\mathbf{r}^{\prime}\right|\right),
$$

where $H_{0}^{(1)}(\bullet)$ is the Hankel function of the first kind and zero order. With a known Green's function in the equation (33), the solution of equation (32) is found by multiplying Green's function by the negative of the source term in equation (33) and integrating the region in which $M(\mathbf{r})$ is different from zero:

$$
P_{S}(\mathbf{r})=-\kappa_{0}^{2} \int_{A} G\left(\mathbf{r} \mid \mathbf{r}^{\prime}\right) M\left(\mathbf{r}^{\prime}\right)\left[P_{I}\left(\mathbf{r}^{\prime}\right)+P_{S}\left(\mathbf{r}^{\prime}\right)\right] d \mathbf{r}^{\prime}
$$

This solution, based on Green's function, is known as the Lippmann-Schwinger integral equation (Lo \& Inderwiesen, 1994). However, this equation presents a nonlinear relationship that is based on the fact that the scattered wavefield $P_{S}(\mathbf{r})$ is present within the integral of the Lippmann-Schwinger integral equation.

The Born approximation is one way to linearize the LippmannSchwinger integral equation and involves assuming that the amplitude of the scattered wavefield $P_{S}(\mathbf{r})$ is much smaller than the incident wavefield $P_{I}(\mathbf{r})$ :

$$
P_{S}(\mathbf{r}) \ll P_{I}(\mathbf{r}) .
$$


Hence, we can simplify equation (35) by considering the following approximation:

$$
P_{S}(\mathbf{r})+P_{I}(\mathbf{r}) \approx P_{I}(\mathbf{r}) .
$$

Therefore, the Lippmann-Schwinger equation can be approximated as follows:

$$
P_{S}(\mathbf{r}) \approx-\kappa_{0}^{2} \int_{A} G\left(\mathbf{r} \mid \mathbf{r}^{\prime}\right) M\left(\mathbf{r}^{\prime}\right) P_{I}\left(\mathbf{r}^{\prime}\right) d \mathbf{r}^{\prime} .
$$

The data function $P_{S}(\mathbf{r})$ and the model function $M(\mathbf{r})$ are now linearly related. Because the primary source is a negative impulse located at $\mathbf{r}_{s}$, we can write the incident wavefield using Green's function (Lo \& Inderwiesen, 1994):

$$
P_{I}(\mathbf{r})=G\left(\mathbf{r} \mid \mathbf{r}_{s}\right)
$$

Substituting equation (39) into equation (38), we obtain the following:

$$
P_{S}\left(\mathbf{r}_{s}, \mathbf{r}_{r}\right) \approx-\kappa_{0}^{2} \int_{A} M\left(\mathbf{r}^{\prime}\right) G\left(\mathbf{r}^{\prime} \mid \mathbf{r}_{s}\right) G\left(\mathbf{r}_{r} \mid \mathbf{r}^{\prime}\right) d \mathbf{r}^{\prime}
$$

where $P_{S}\left(\mathbf{r}_{s}, \mathbf{r}_{r}\right)$ is the scattered wavefield registered at $\mathbf{r}_{r}$. Equation (40) shows the Lippmann-Schwinger integral equation linearized through the Born approximation. However, the Born approximation requires a weak scattering; in other words, there should be a low velocity contrast.

\section{Diffraction Tomography Matrix Formulation}

As observed above, the Born approximation allows us to write a linear relationship between the scattered field $P_{S}\left(\mathbf{r}_{s}, \mathbf{r}_{r}\right)$ and the model function $M(\mathbf{r})$ :

$$
P_{S}\left(\kappa, \mathbf{r}_{s}, \mathbf{r}_{r}\right) \approx-\kappa^{2} \int_{A} M\left(\mathbf{r}^{\prime}\right) G\left(\mathbf{r}^{\prime} \mid \mathbf{r}_{s}\right) G\left(\mathbf{r}_{r} \mid \mathbf{r}^{\prime}\right) d \mathbf{r}^{\prime},
$$

where $G\left(\mathbf{r}^{\prime} \mid \mathbf{r}_{s}\right)$ is Green's function expressed as a Hankel function of the first kind and zero order. Substituting equation (34) into equation (41), we obtain the following:

$$
\begin{aligned}
& P_{S}\left(\kappa, \mathbf{r}_{s}, \mathbf{r}_{r}\right) \approx \frac{\kappa^{2}}{16} \int_{A} M\left(\mathbf{r}^{\prime}\right) H_{0}^{(1)} \\
& \times\left(\kappa\left|\mathbf{r}^{\prime}-\mathbf{r}_{s}\right|\right) H_{0}^{(1)}\left(\kappa\left|\mathbf{r}_{r}-\mathbf{r}^{\prime}\right|\right) d \mathbf{r}^{\prime} .
\end{aligned}
$$

It is necessary to establish a means to characterize the velocity that is continuous in space and uses a finite number of coefficients (Rocha Filho et al., 1996). As in traveltime tomography, we parameterize the area through a finite number of blocks $I$. Because the seismic velocity is constant inside each block, the object function $m_{i}$ will also be constant within each block:

$$
M(\mathbf{r})=\sum_{i=1}^{I} m_{i} \phi_{i}\left(\mathbf{r}^{\prime}\right),
$$

where $\phi_{i}\left(\mathbf{r}^{\prime}\right)$ is a basis function. Our basis function is defined as $\phi_{i}\left(\mathbf{r}^{\prime}\right)=1$ when $\mathbf{r}^{\prime}$ is within the area $i$, and $\phi_{i}\left(\mathbf{r}^{\prime}\right)=0$ when it is outside the area. Substituting equation (43) in the equation (41), we obtain the following:

$$
\begin{gathered}
P_{S}\left(\kappa, \mathbf{r}_{s}, \mathbf{r}_{r}\right) \approx \frac{\kappa^{2}}{16} \int_{A} \sum_{i=1}^{I} m_{i} \phi_{i}\left(\mathbf{r}^{\prime}\right) \times \\
H_{0}^{(1)}\left(\kappa\left|\mathbf{r}^{\prime}-\mathbf{r}_{s}\right|\right) H_{0}^{(1)}\left(\kappa\left|\mathbf{r}_{\mathrm{r}}-\mathbf{r}^{\prime}\right|\right) d \mathbf{r}^{\prime} .
\end{gathered}
$$

There are $M$ sources located at $\mathbf{r}_{s m}(1 \leq m \leq M)$ and $N$ receptors located at $\mathbf{r}_{r n}(1 \leq n \leq N)$. In our multi-frequency approach, there are $L$ wavenumbers $\kappa(1 \leq l \leq L)$, which produces the following equation:

$$
\begin{gathered}
P_{S}\left(\kappa_{l}, \mathbf{r}_{s m}, \mathbf{r}_{r n}\right) \approx \frac{\kappa^{2}}{16} \int_{A} \sum_{i=1}^{I} m_{i} \phi_{i}\left(\mathbf{r}^{\prime}\right) \times \\
H_{0}^{(1)}\left(\kappa\left|\mathbf{r}^{\prime}-\mathbf{r}_{s m}\right|\right) H_{0}^{(1)}\left(\kappa\left|\mathbf{r}_{r n}-\mathbf{r}^{\prime}\right|\right) d \mathbf{r}^{\prime} .
\end{gathered}
$$

We use $W$ as an auxiliary variable:

$$
\begin{gathered}
W_{l m n i}= \\
\frac{\kappa^{2}}{16} \int_{A} \phi_{i}\left(\mathbf{r}^{\prime}\right) H_{0}^{(1)}\left(\kappa_{l}\left|\mathbf{r}^{\prime}-\mathbf{r}_{s m}\right|\right) H_{0}^{(1)}\left(\kappa\left|\mathbf{r}_{r n}-\mathbf{r}^{\prime}\right|\right) d \mathbf{r}^{\prime} .
\end{gathered}
$$

Therefore, the scattered field is discretized so that the cells are much smaller than the wavelength of the incident wavefield, and the continuous integral is substituted by a 2-D sum in space:

$$
\begin{gathered}
W_{l m n i}=\frac{\kappa^{2}}{16} \sum_{A} \phi_{i}\left(x^{\prime}, z^{\prime}\right) H_{0}^{(1)}\left(\kappa_{l} \mid\left(x^{\prime}, z^{\prime}\right)\right. \\
\left.-\left(x_{s m}, z_{s m}\right) \mid\right) H_{0}^{(1)}\left(\kappa_{l}\left|\left(x^{\prime}, z^{\prime}\right)-\left(x_{r n}, z_{r n}\right)\right|\right) \Delta x \Delta z .
\end{gathered}
$$

Then, we rewrite the initial problem with a system of linear equations:

$$
P_{s, l m n}=\sum_{i=1}^{I} W_{l m n i} m_{i},
$$

which can be written in matrix notation as

$$
\mathbf{P}=\mathbf{W m}
$$

For the wavefield propagation in our seismic numerical experiment, we used the finite difference method. The modeling consists of the use of discrete approximations of the space and time derivatives of the wave equation. In this study, we used the second-order approximation for time and the fourth-order approximation for space. 


\section{Gassmann's Equation, Fluids and Carbon Dioxide}

Gassmann's equation (Gassmann, 1951) is a valuable tool in the study of reservoirs because it allows the possibility of modeling several scenarios with different fluid types and fluid saturations. The equation relates the bulk modulus $K$ of a given rock to the pore, frame and fluid properties:

$$
K_{\text {sat }}=K_{d r y}+\frac{\left(1-\frac{K_{d r y}}{K_{\min }}\right)^{2}}{\frac{\phi}{K_{\text {fluid }}}+\frac{(1-\phi)}{K_{\min }}-\frac{K_{d r y}}{K_{\min }^{2}}},
$$

where $K_{\text {sat }}$ is the bulk modulus of the saturated rock, $K_{\text {fluid }}$ is the bulk modulus of the fluid, $K_{d r y}$ is the bulk modulus of the dry rock, $K_{\min }$ is the bulk modulus of the mineral and $\phi$ is the rock porosity. The shear modulus is not affected by the presence of fluids; thus,

$$
\mu_{\text {sat }}=\mu_{d r y},
$$

where $\mu_{\text {sat }}$ is the shear modulus of the saturated rock and $\mu_{d r y}$ is the shear modulus of the dry rock. Gassmann's equation is based on various assumptions (Wang, 2001): (i) The rock is uniform and isotropic, and the pore space is completely connected; (ii) the rock is composed of a single type of mineral; (iii) the equation is valid for low frequencies; and (iv) the fluid does not interact with the rock. Smith et al. (2003) showed that the bulk modulus of a rock composed of a single type of mineral can be calculated from the Hill's average.

From the bulk modulus of the saturated rock modeled with the Gassmann's equation, we can estimate the velocity of the saturated reservoir with any type of fluid:

$$
V_{p}=\sqrt{\frac{K_{\text {sat }}+\frac{4}{3} \mu}{\rho} .}
$$

The main purpose of reservoir characterization and monitoring is to map and delineate the distribution of each fluid type in the porous media. Thus, to succeed, the seismic data should have high-enough resolution to image different compressibility values within the reservoir (Wang, 2001). Therefore, a thorough understanding of the seismic properties of the porous media is important.

Saline reservoirs are a possible target for $\mathrm{CO}_{2}$ injection, and this technique, carbon capture and storage, has been applied successfully in various parts of the world. However, the $\mathrm{CO}_{2}$ injection for storage purposes requires planning the operating techniques and predicting the behavior of the system, which involves both rock and fluids. Schiitt et al. (2005) presented several questions that must be answered before the injection process: (i) What is the long-term fate of the $\mathrm{CO}_{2}$ and how will it mix with the brine? (ii) Will the $\mathrm{CO}_{2}$ precipitate or will it dissolve the reservoir minerals, changing the original properties and interfering with the geomechanical system? (iii) Can geophysical methods provide reliable data on the saturation and reservoir pressure estimates? To safely inject $\mathrm{CO}_{2}$, feasibility studies must address these questions. In this context, the current work is presented as a study of the feasibility of diffraction tomography as a tool for monitoring injected $\mathrm{CO}_{2}$.

\section{Numerical Simulations}

The model used in this study corresponds to a situation in which a reservoir is completely saturated with water. We used the method of finite differences to model the wavefield recorded at the receivers. The medium illustrated in Figure 1 was parameterized in a $30 \times 30$ grid, resulting in 900 blocks. The acquisition geometry used is well-to-well; 15 sources are located in the left-side borehole, and 30 receivers are located in the right-side borehole. This configuration would result in 450 equations, but the values of the scattered field are complex; thus, we separated the real and imaginary parts. For each frequency, we have 900 (2 times 450) data parameters or equations. We employed the multi-frequency approach to obtain more data. For this set of simulations, we used four frequencies: 90, 105, 120 and $135 \mathrm{~Hz}$. Thus, in the matrix A, $N=900$, and $M=3600$.

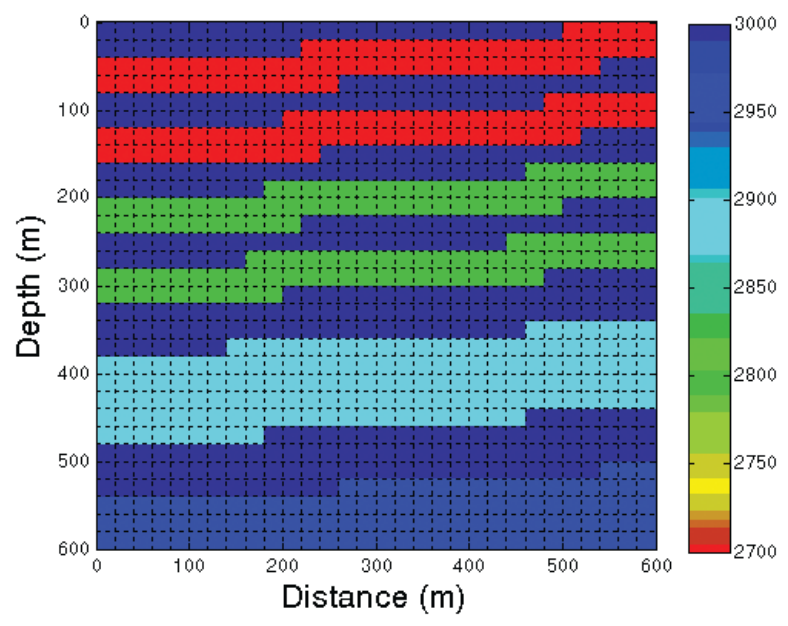

Figure 1 - First stage true model parameters with $100 \%$ of water saturation, before the injection of $\mathrm{CO}_{2}$ in the reservoir. The color bar represents the P-wave velocity in $\mathrm{m} / \mathrm{s}$.

As discussed previously, inverse problems are generally illposed, and diffraction tomography is not an exception. The illconditioned nature of the inverse problem is analysed using two methods: (i) the selection of singular values, where the optimum 
Table 1 - Reservoir properties before injection, where $K_{d r y}$ is the bulk modulus of the dry rock, $K_{q u a r}$ is the bulk modulus of quartz, $K_{\text {clay }}$ is the bulk modulus of clay, $K_{\text {feld }}$ is the bulk modulus of feldspar, $\rho_{\text {quar }}$ is the density of quartz, $\rho_{\text {clay }}$ is the density of clay, $\rho_{\text {feld }}$ is the density of feldspar, $\phi$ is the porosity and $\mu$ is the shear modulus. The values of different bulk moduli and the shear modulus are given in GPa and the densities in $\mathrm{g} / \mathrm{cm}^{3}$.

\begin{tabular}{|c|c|c|c|c|c|c|c|c|}
\hline \multicolumn{10}{|c|}{ Static reservoir properties } \\
\hline$K_{\text {dry }}$ & $K_{\text {quar }}$ & $K_{\text {clay }}$ & $K_{\text {feld }}$ & $\rho_{\text {quar }}$ & $\rho_{\text {clay }}$ & $\rho_{\text {feld }}$ & $\phi$ & $\mu$ \\
\hline 7.4 & 10.5 & 19.8 & 30.6 & 2.65 & 2.58 & 2.63 & 0.22 & 4.2 \\
\hline
\end{tabular}

Table 2 - Reservoir properties during injection, where $\mathrm{S}_{\mathrm{H} 2 \mathrm{O}}$ is the water saturation, $\mathrm{S}_{\mathrm{CO} 2}$ is the $\mathrm{CO}_{2}$ saturation, $K_{\mathrm{CO} 2}$ is the bulk modulus of $\mathrm{CO}_{2}, K_{\text {sat }}$ is the bulk modulus of the saturated rock, $\rho_{\text {sat }}$ is the density of the saturated rock and $V_{p}$ is the P-wave velocity. The values of different bulk moduli are given in $\mathrm{GPa}$, the densities in $\mathrm{g} / \mathrm{cm}^{3}$ and the P-wave velocity in $\mathrm{m} / \mathrm{s}$. The water density is $1 \mathrm{~g} / \mathrm{cm}^{3}$, and the bulk modulus of water is $2.25 \mathrm{GPa}$.

\begin{tabular}{|c|c|c|c|c|c|c|}
\hline \multicolumn{7}{|c|}{ Reservoir properties during injection } \\
\hline$S_{\mathrm{H} 2 \mathrm{O}}$ & $S_{\mathrm{CO} 2}$ & $K_{\mathrm{CO} 2}$ & $K_{\text {sat }}$ & $\rho_{\mathrm{CO} 2}$ & $\rho_{\text {sat }}$ & $V_{p}$ \\
\hline $100 \%$ & $0 \%$ & 0.25 & 13.60 & 0.71 & 2.27 & 2900 \\
\hline $70 \%$ & $30 \%$ & 0.25 & 9.43 & 0.71 & 2.25 & 2584 \\
\hline $40 \%$ & $60 \%$ & 0.25 & 8.62 & 0.71 & 2.23 & 2523 \\
\hline
\end{tabular}

number is determined by energy and entropy criteria, and (ii) the regularization by derivative matrices, where the optimum parameter is chosen using generalized cross correlation. To evaluate the robustness of the algorithm, we added three levels of Gaussian noise to the scattered wavefield: $0.05,0.10$ and 0.15 , where these numbers represent the amplitude of the quasi-random sequence. The estimated tomograms shown in this paper were obtained when the scattered field was corrupted with the factor 0.05 .

Figure 1 shows the geological model considered in this work. The model includes six sandstone reservoirs that are fully saturated with water and separated by layers of shale. $\mathrm{CO}_{2}$ was injected into the fifth layer from the top. This layer is considered to be composed of quartz, feldspar and clay in proportions of $65 \%$, $20 \%$ and $15 \%$, respectively. Other minerals have been ignored for simplicity. Table 1 shows the main parameters of the reservoir. The first stage is the pre-injection stage, which is very important in understanding the geological context of the area and, in conjunction with subsequent stages results, can be used to track the path of the gas within the layer.

In the subsequent stages, we considered different water and $\mathrm{CO}_{2}$ saturation levels within the reservoir. Table 2 shows the saturation values considered for these simulations, as well as their influence on the elastic parameters.

The multi-frequency approach resulted in the following results for the first stage: Figure 2 shows the energy of the estimated model parameters, and Figure 3 shows the entropy of the estimated model parameters. Both curves feature a region of max- imum stability, in which the energy and entropy become unstable. Figure 4 shows the RMS error between the true and estimated model parameters, which confirms the existence of a critical point in these two figures. The optimal quantity was found to be 545 singular values. Figure 5 shows the estimated model parameters. Figure 6 shows the curve of the GCV, and the optimal regularization parameter that minimizes the GCV curve corresponds to $10^{-1}$. Figure 7 presents the estimated model parameters with regularization by derivative matrices for $\lambda=10^{-1}$.

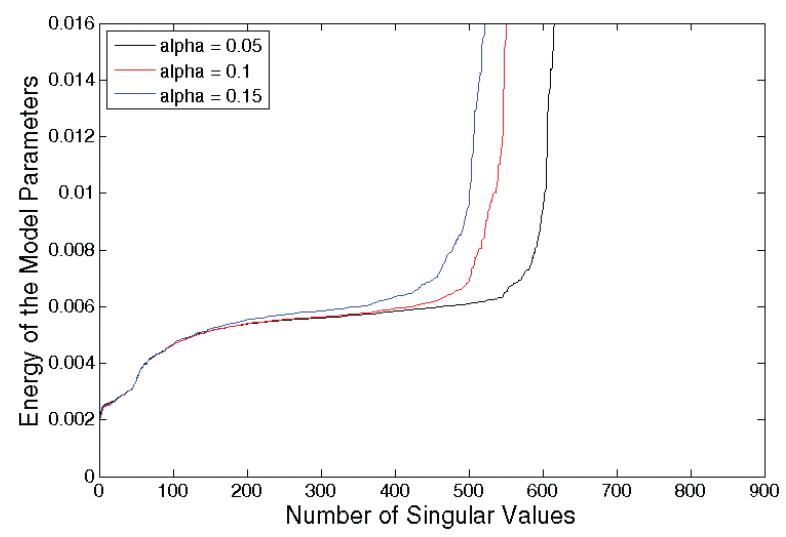

Figure 2 - First stage energy of the estimated model parameters, as a function of quantity of singular values used to compute the pseudo-inverse matrix. Each curve represents a different noise level added to the scattered field.

For the second stage, we assigned the aquifer a distribution of $0 \%$ gas and $100 \%$ water. Figure 8 illustrates the ultimate distribution of water and gas in the reservoir. Notice that there is a 


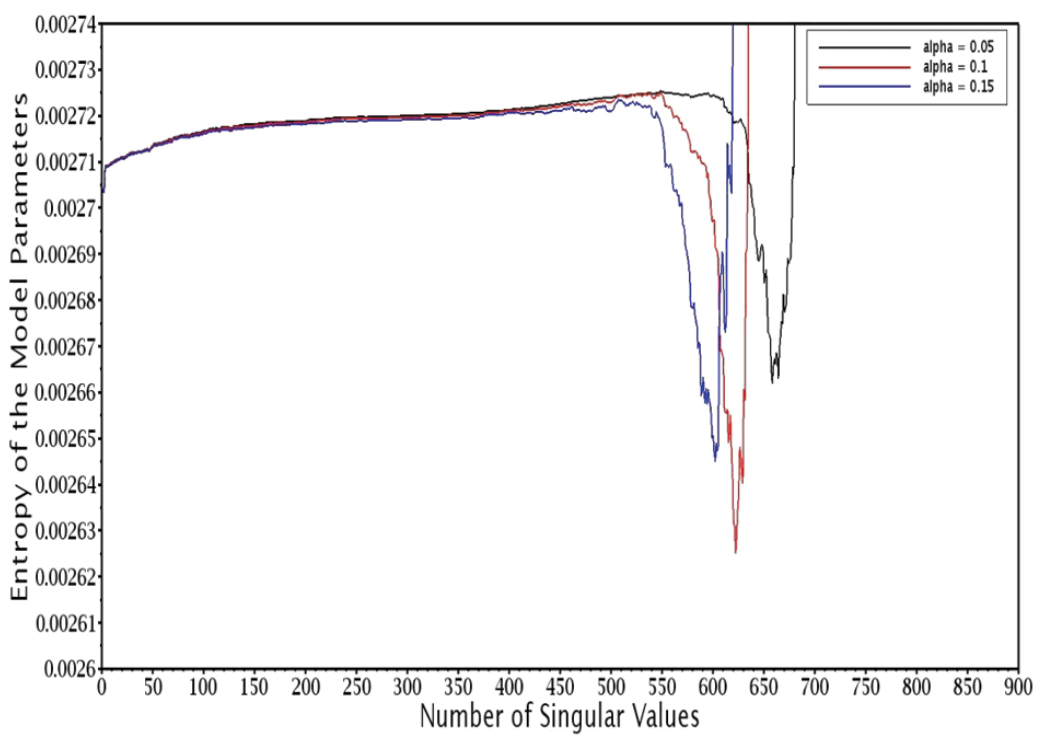

Figure 3 - First stage entropy of the estimated model parameters, as a function of quantity of singular values used to compute the pseudo-inverse matrix. Each curve represents a different noise level added to the scattered field.

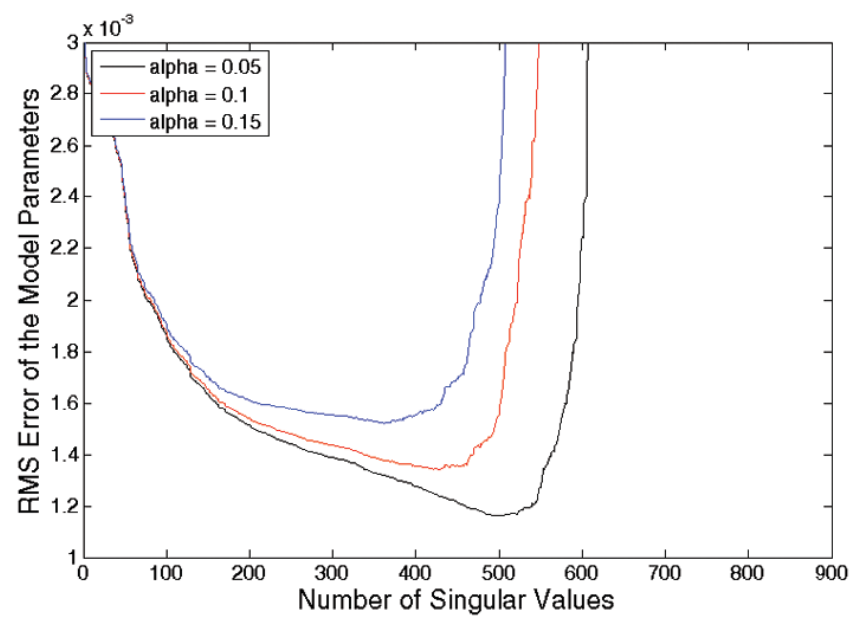

Figure 4 - First stage RMS error between true and estimated model parameters, as a function of quantity of singular values used to compute the pseudo-inverse matrix. Each curve represents a different noise level added to the scattered field.

smaller part of the layer (in red) with 30\% gas and $70 \%$ water. The velocity values for the two types of saturations are shown in Table 2. We chose 650 singular values (figures not shown due to space limitations). Figure 9 shows the estimated model parameters, and Figure 10 shows the residual model parameters between stages 1 and 2, which are used to analyze the fluid flow behavior. The GCV curve for the second stage, not shown, indicates that the optimal parameter cutoff is again $10^{-1}$. Figure 11 shows the estimated model with the optimal regularization parameter. Figure 12 shows the residual estimated model parameters between stages 1 and 2. Figure 12 is smoother that Figure 10, which is not surprising because this is characteristic of the regularization method.

For the third stage, besides the two conditions already described for Figure 8, a portion of the layer presents a third condition, with $60 \%$ gas and $40 \%$ water. Figure 13 illustrates the distribution of water and gas in the reservoir. The velocity values for the three types of saturations are shown in Table 2. The curves indicated 580 singular values (figures not shown). Figure 14 shows the estimated model parameters. Figures 15 and 16 show the residual model parameters between stages 1 and 3 and between stages 2 and 3 , respectively. 


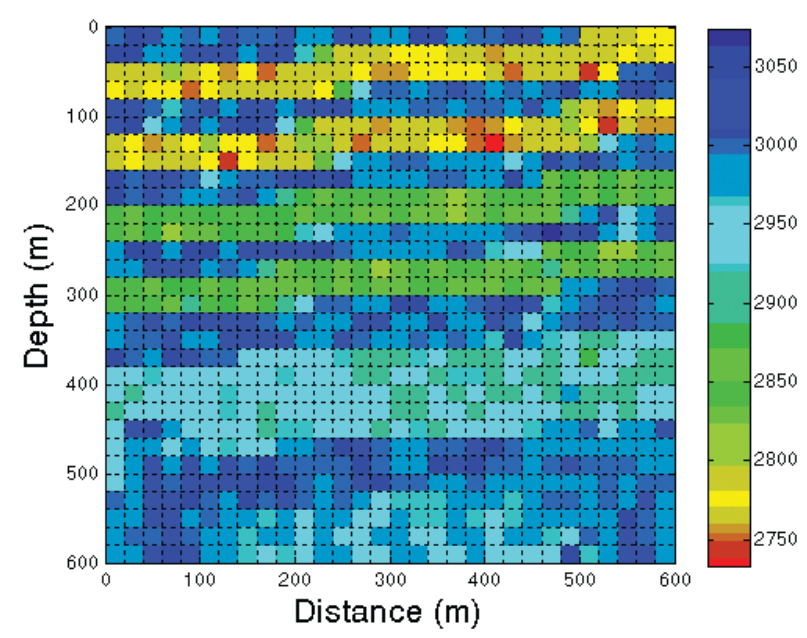

Figure 5 - First stage estimated model parameters by selection of singular values, using 545 singular values. The color bar represents the P-wave velocity in $\mathrm{m} / \mathrm{s}$.

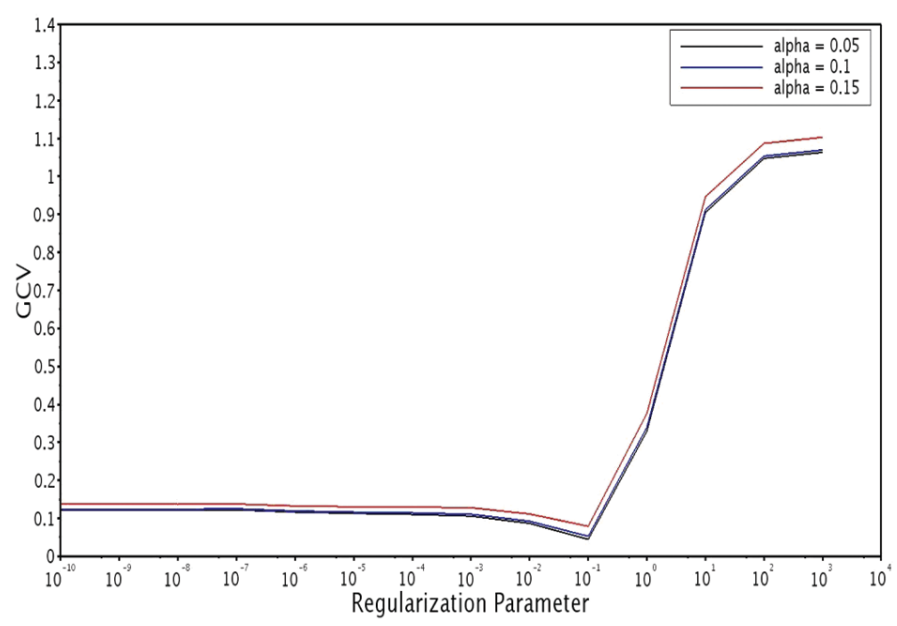

Figure 6 - First stage GCV curve as a function of the regularization parameter. Each curve represents a different noise level added to the scattered field.

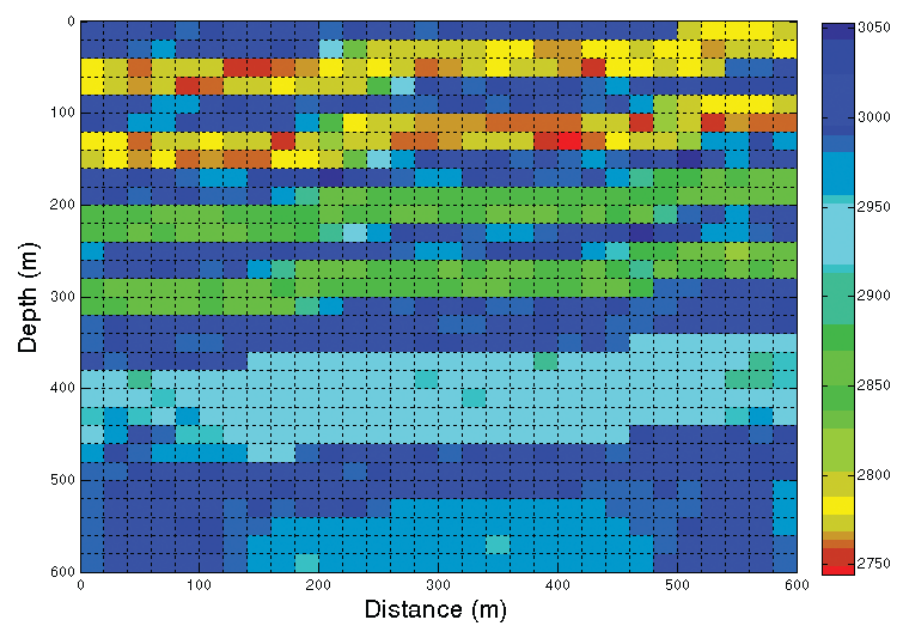

Figure 7 - First stage estimated model parameters via regularization by derivative matrices; regularization parameter $\lambda=10^{-1}$. The color bar represents the P-wave velocity in $\mathrm{m} / \mathrm{s}$. 


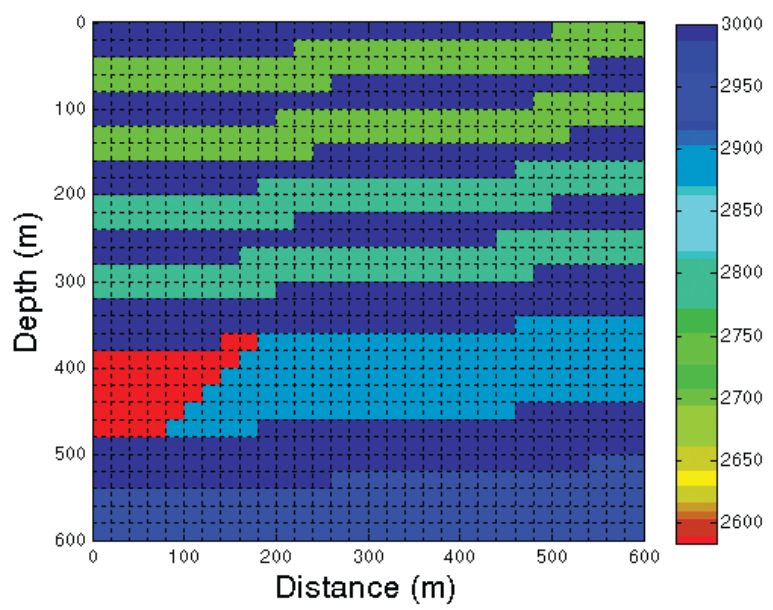

Figure 8 - Second stage true model parameters with different levels of water and $\mathrm{CO}_{2}$ saturation in the reservoir. The color bar represents the P-wave velocity in $\mathrm{m} / \mathrm{s}$.

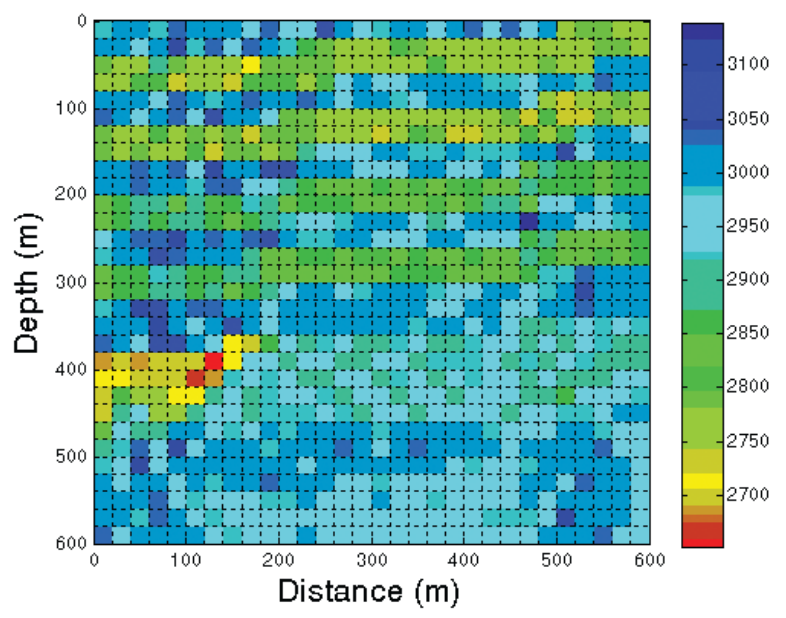

Figure 9 - Second stage estimated model parameters by selection of singular values, using 650 singular values. The color bar represents the P-wave velocity in $\mathrm{m} / \mathrm{s}$.

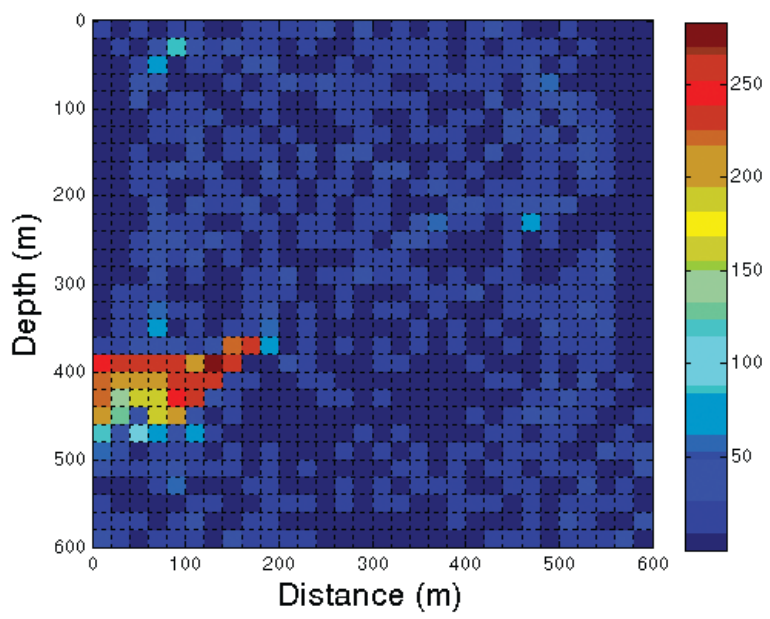

Figure 10 - Residual estimated model parameters between the first and second stages by selection of singular values. The color bar represents the P-wave velocity in $\mathrm{m} / \mathrm{s}$. 


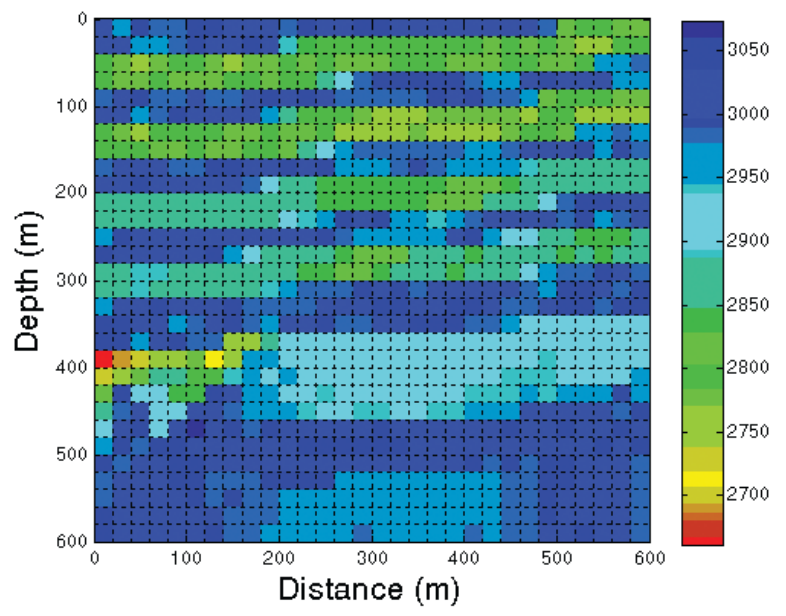

Figure 11 - Second stage estimated model parameters via regularization by derivative matrices; regularization parameter $\lambda=10^{-1}$. The color bar represents the P-wave velocity in $\mathrm{m} / \mathrm{s}$.

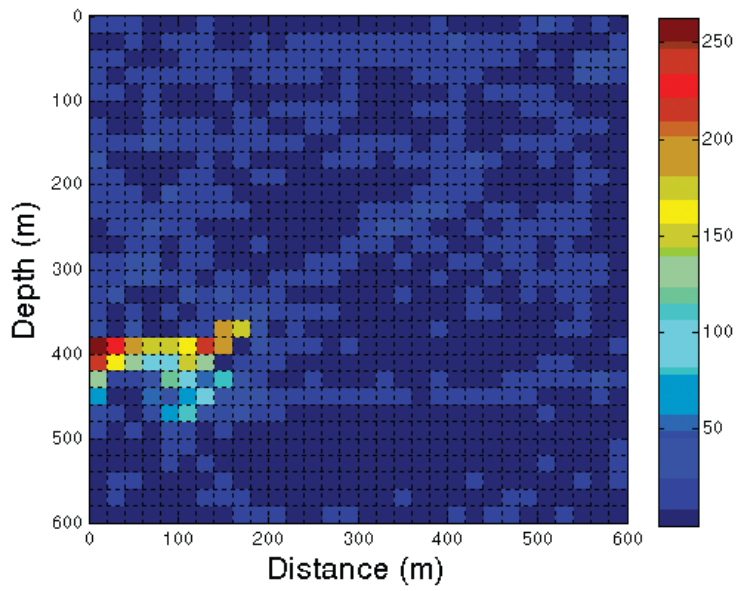

Figure 12 - Residual estimated model parameters between the first and second stages via regularization by derivative matrices. The color bar represents the P-wave velocity in $\mathrm{m} / \mathrm{s}$.

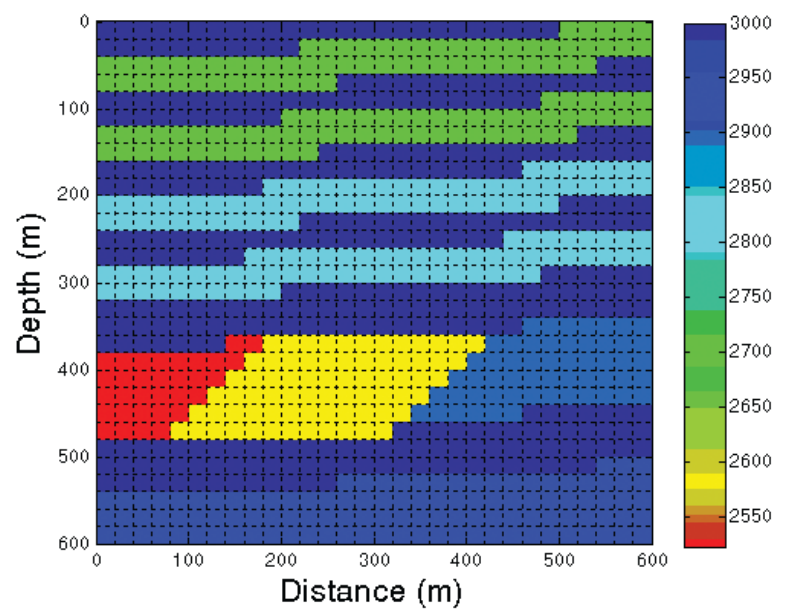

Figure 13 - Third stage true model parameters with different levels of water and $\mathrm{CO}_{2}$ saturation in the reservoir. The color bar represents the P-wave velocity in $\mathrm{m} / \mathrm{s}$. 


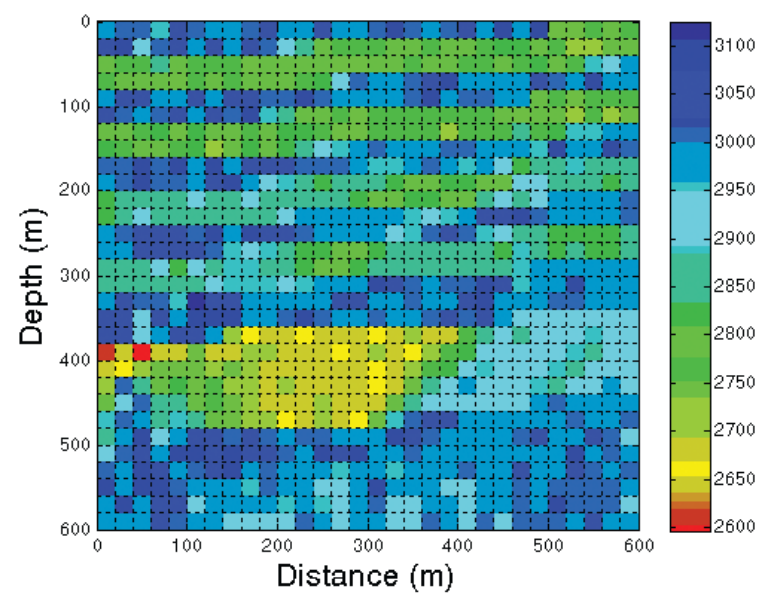

Figure 14 - Third stage estimated model parameters by selection of singular values, using 580 singular values. The color bar represents the P-wave velocity in $\mathrm{m} / \mathrm{s}$.

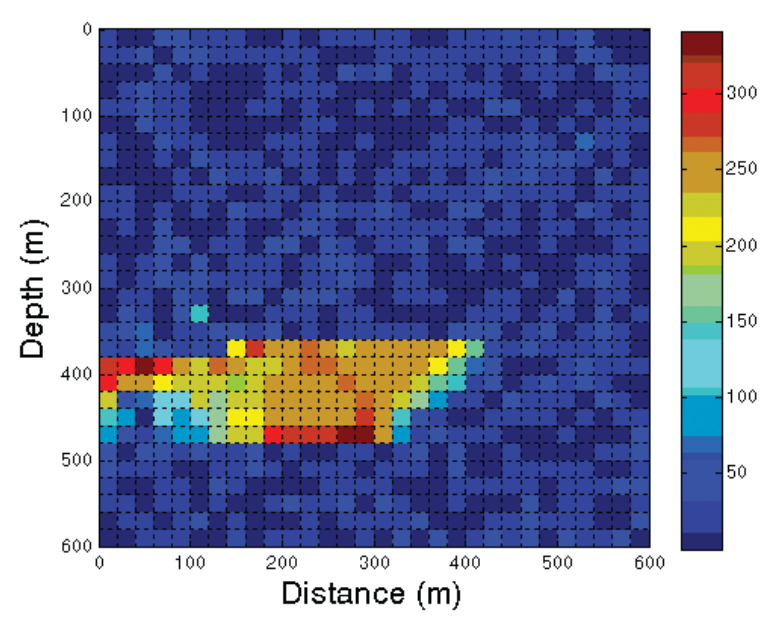

Figure 15 - Residual estimated model parameters between the first and third stages by selection of singular values. The color bar represents the P-wave velocity in $\mathrm{m} / \mathrm{s}$.

The GCV curve for the third stage, again not shown, indicates that the optimal parameter cutoff is also $10^{-1}$. Figure 17 shows the estimated model with the optimal regularization parameter. Figures 18 and 19 show the residual model parameters between stages 1 and 3 and between stages 2 and 3 , respectively. Figure 18 is very similar to Figure 15, and Figure 19 is very similar to Figure 16.

Finally, using the matrix multi-frequency approach, we analyzed the results of the last stage, in which the aquifer was assigned a distribution of $60 \%$ gas and $40 \%$ water (illustrated in Figure 20). Figure 21 shows the estimated model parameters obtained by the selection of singular values (620 singular values). We then compared the last stage to all the others: Figure 22 shows the residual model parameters between stages 1 and 4 ,
Figure 23 between stages 2 and 4, and Figure 24 between stages 3 and 4. The GCV curve for the fourth and final stage indicates that the optimal parameter cutoff is also $10^{-1}$. Figure 25 shows the estimated model with the optimal regularization parameter. Additionally, the last stage was also compared to all the others for the regularization approach: Figure 26 shows the residual model parameters between stages 1 and 4, Figure 27 between stages 2 and 4 , and Figure 28 between stages 3 and 4 . As before, we compared the images provided by the two methodologies: Figure 22 was compared to Figure 26, Figure 23 to Figure 27, and Figure 24 to Figure 28. The results are quite similar, and the figures from the regularization method are smoother. However, in the comparison of stages 3 and 4 , the selection of singular values provides a cleaner image. 


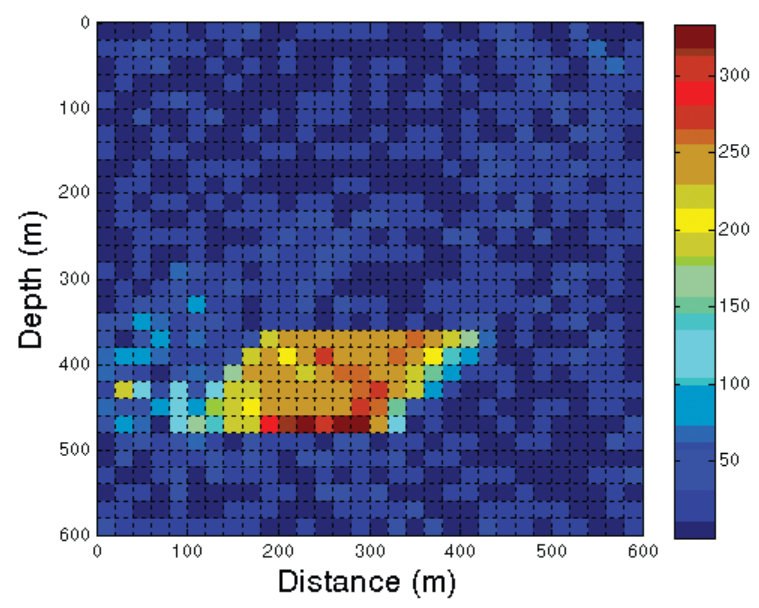

Figure 16 - Residual estimated model parameters between the second and third stages by selection of singular values. The color bar represents the P-wave velocity in $\mathrm{m} / \mathrm{s}$.

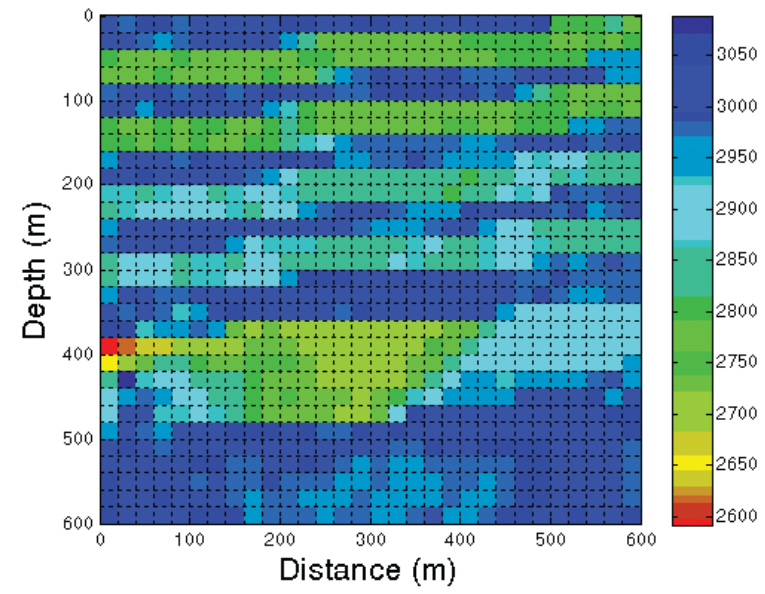

Figure 17 - Third stage estimated model parameters via regularization by derivative matrices; regularization parameter $\lambda=10^{-1}$. The color bar represents the P-wave velocity in $\mathrm{m} / \mathrm{s}$.

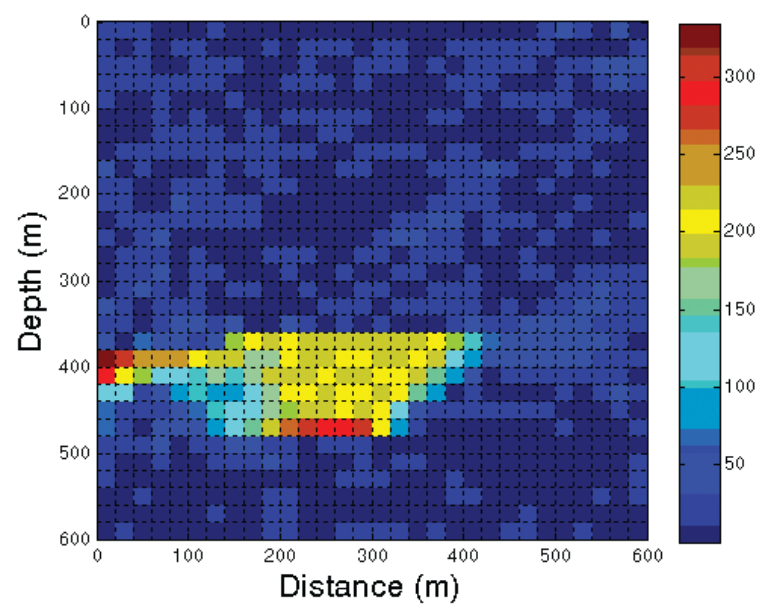

Figure 18 - Residual estimated model parameters between the first and third stages via regularization by derivative matrices. The color bar represents the P-wave velocity in $\mathrm{m} / \mathrm{s}$. 


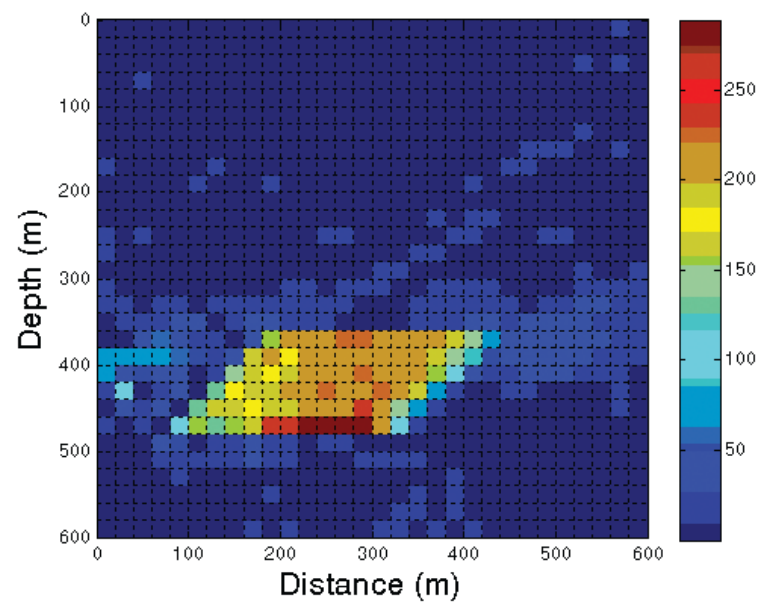

Figure 19 - Residual estimated model parameters between the second and third stages via regularization by derivative matrices. The color bar represents the P-wave velocity in $\mathrm{m} / \mathrm{s}$.

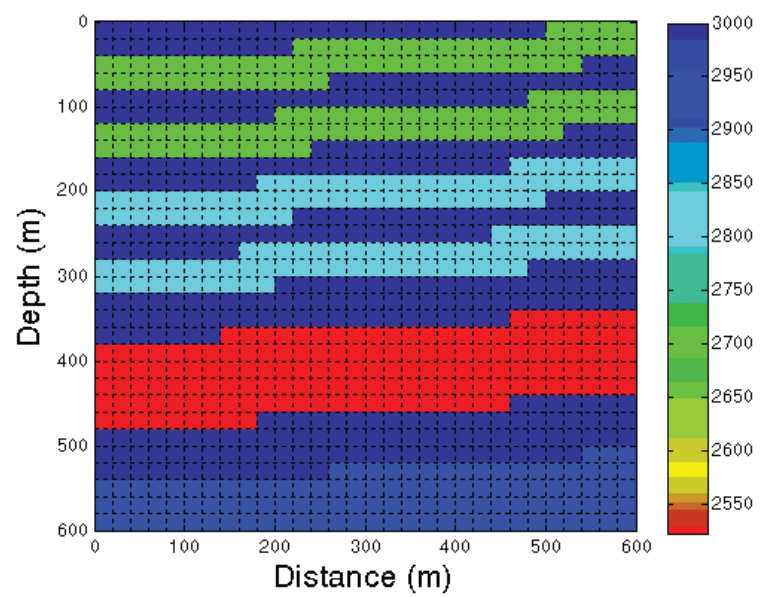

Figure 20 - Fourth stage true model parameters with different levels of water and $\mathrm{CO}_{2}$ saturation in the reservoir. The color bar represents the P-wave velocity in $\mathrm{m} / \mathrm{s}$.

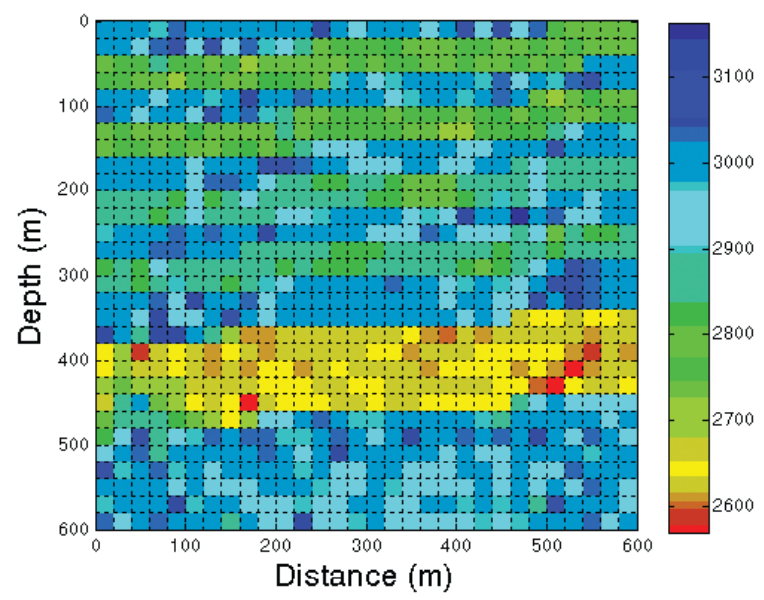

Figure 21 - Fourth stage estimated model parameters by selection of singular values, using 620 singular values. The color bar represents the P-wave velocity in $\mathrm{m} / \mathrm{s}$. 


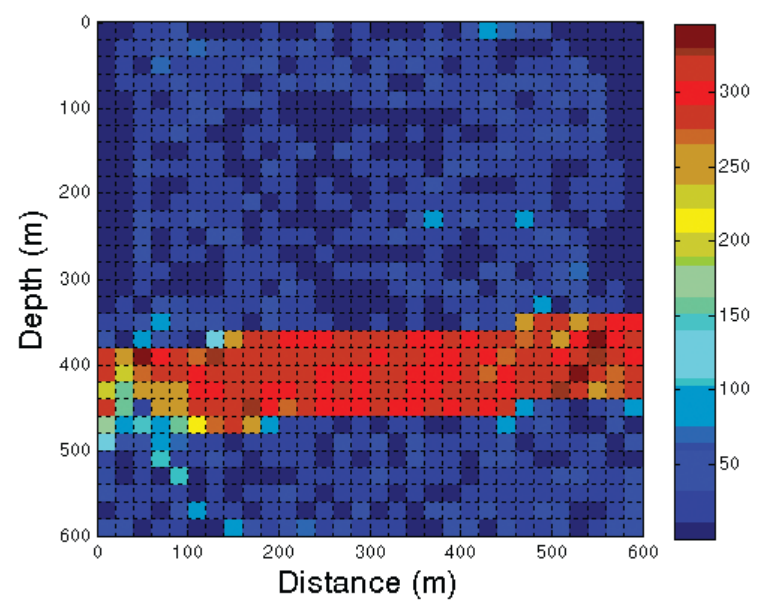

Figure 22 - Residual estimated model parameters between the first and fourth stages by selection of singular values. The color bar represents the P-wave velocity in $\mathrm{m} / \mathrm{s}$.

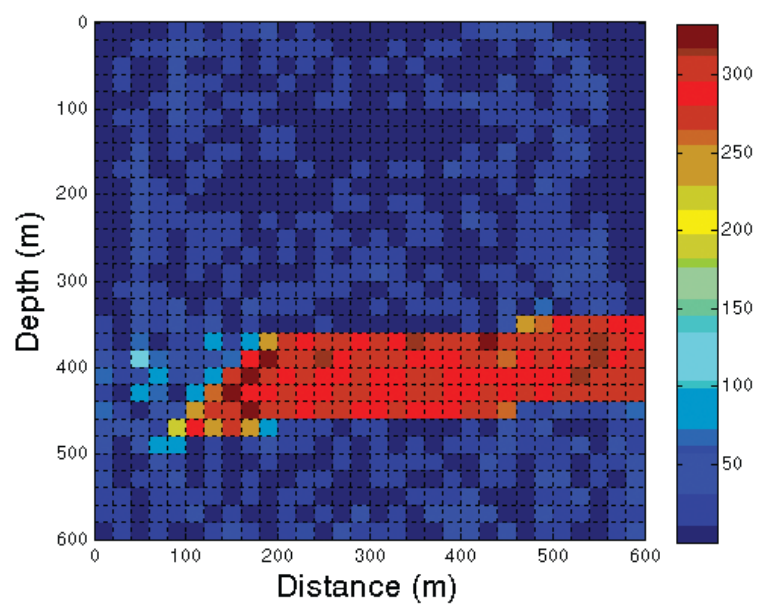

Figure 23 - Residual estimated model parameters between the second and fourth stages by selection of singular values. The color bar represents the P-wave velocity in $\mathrm{m} / \mathrm{s}$.

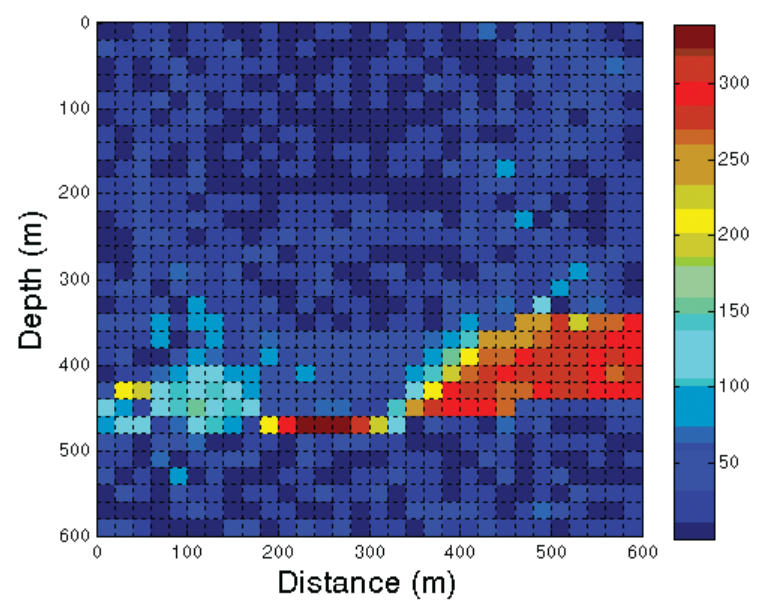

Figure 24 - Residual estimated model parameters between the third and fourth stages by selection of singular values. The color bar represents the P-wave velocity in $\mathrm{m} / \mathrm{s}$. 


\section{CONCLUSIONS}

Gassmann's equation can be used to model the velocity changes associated with a substitution fluid inside of a reservoir. In conjunction with Gassmann's equation modeling results, we used a multi-frequency approach to diffraction tomography, which is an ill-posed inverse problem. To obtain satisfactory results, the ill-posed nature of the problem was minimized by the selection of singular values in the construction of the generalized inverse matrix and by regularization via derivative matrices.

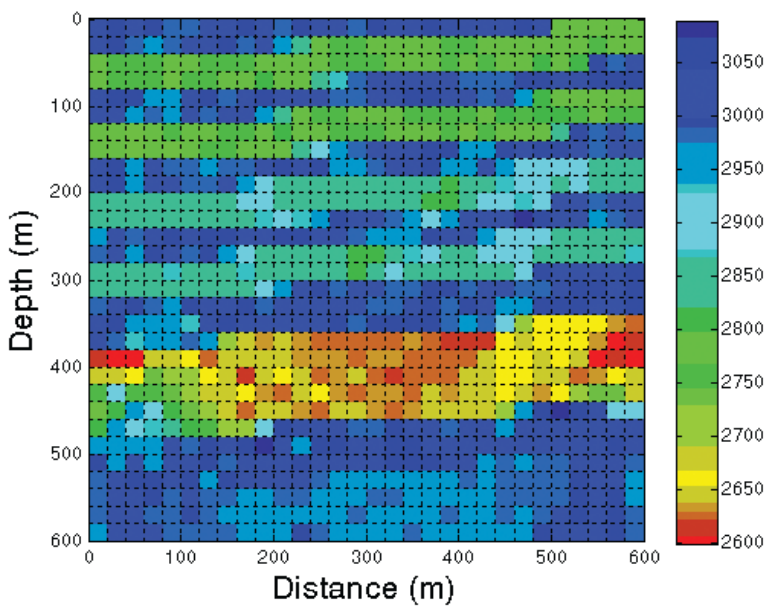

Figure 25 - Fourth stage estimated model parameters via regularization by derivative matrices; regularization parameter $\lambda=10^{-1}$. The color bar represents the P-wave velocity in $\mathrm{m} / \mathrm{s}$.

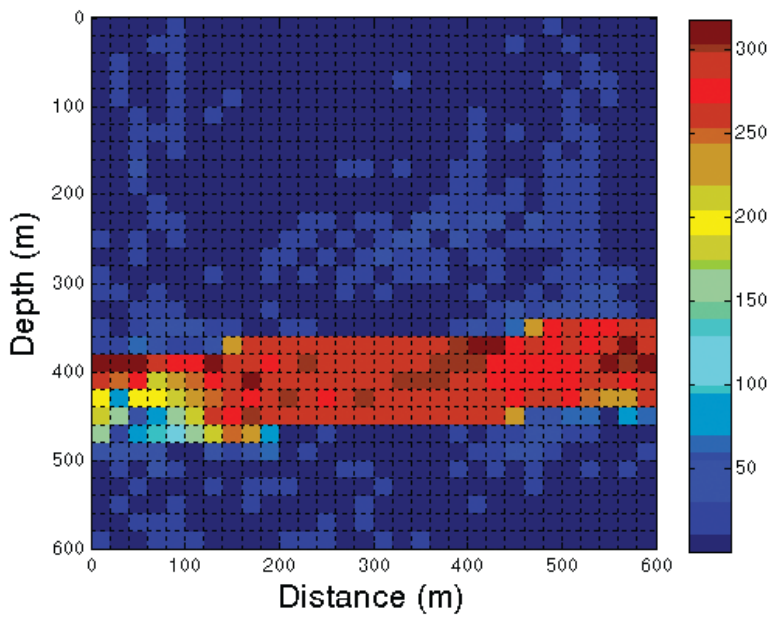

Figure 26 - Residual estimated model parameters between the first and fourth stages via regularization by derivative matrices. The color bar represents the P-wave velocity in $\mathrm{m} / \mathrm{s}$.

Seismic diffraction tomography proved to be a robust tool for monitoring injected $\mathrm{CO}_{2}$ in sandstone reservoirs; fluid migration inside of the reservoir was identified and tracked based on velocity variations detected during several stages of $\mathrm{CO}_{2}$ injection.

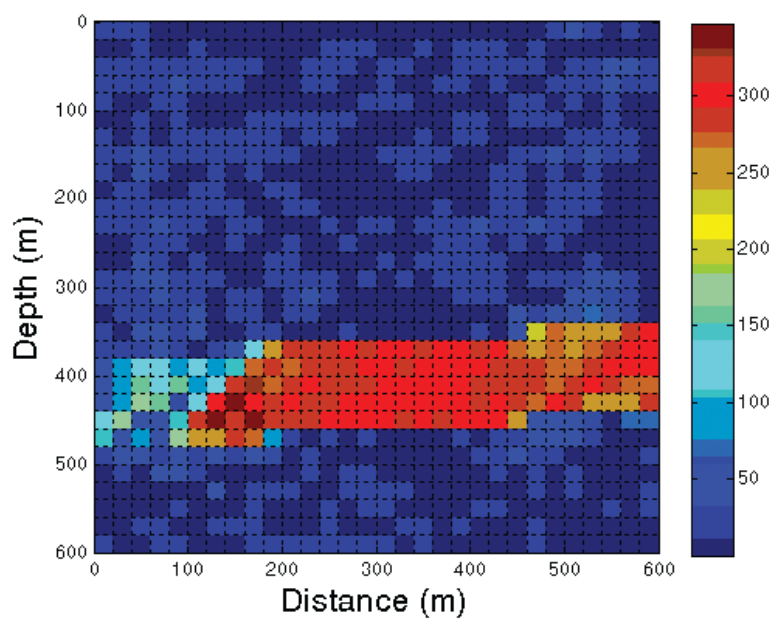

Figure 27 - Residual estimated model parameters between the second and fourth stages via regularization by derivative matrices. The color bar represents the P-wave velocity in $\mathrm{m} / \mathrm{s}$.

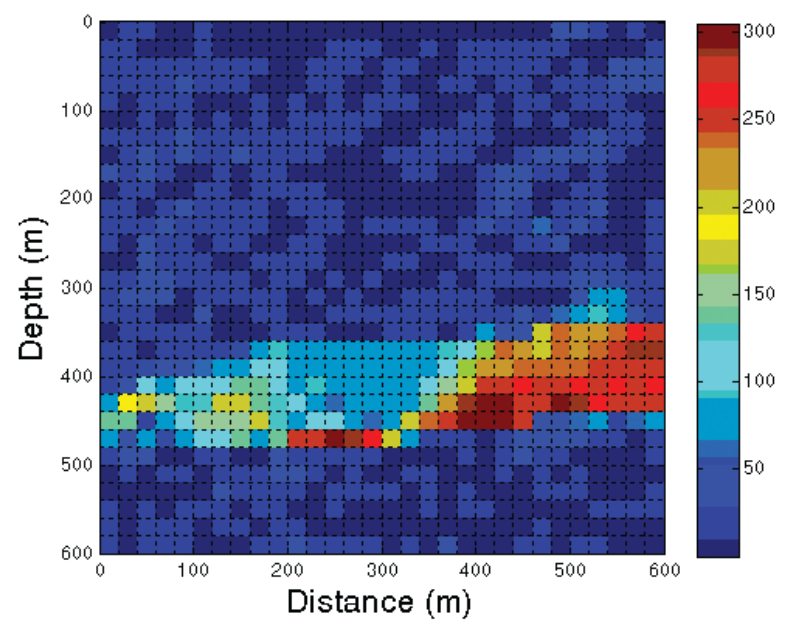

Figure 28 - Residual estimated model parameters between the third and fourth stages via regularization by derivative matrices. The color bar represents the P-wave velocity in $\mathrm{m} / \mathrm{s}$.

Both methods used to address the ill-posed nature of the inverse problem were effective, based on the satisfactory results produced by both the optimal number of singular values and the regularization via derivative matrices.

The energy and entropy criteria for the model parameters were adequate for choosing the optimal number of singular values. This finding was supported by the RMS error between the true and estimated model parameters. This approach establishes the maximum number of singular values to be inserted into the construction of the generalized inverse matrix. For the regularization by derivative matrices technique, the GCV method proved to be an effective approach for choosing the optimal regularization parameter. 


\section{ACKNOWLEDGEMENTS}

The authors thank PETROBRAS for sponsoring the project Seismic Tomography for $\mathrm{CO}_{2}$ Geologic Storage Monitoring. C.J.M.G. Silva would like to thank CNPq for a M.Sc. scholarship. A. Bassrei would like to thank CNPq for the projects National Institute of Science and Technology in Petroleum Geophysics (INCT-GP) and 308690/2013-3 (research fellowship). A. Bassrei would also like to thank FINEP for sponsoring the CTPETRO Network in Exploration Geophysics (Rede 01).

\section{REFERENCES}

BASSREI A \& RODI W. 1993. Regularization and inversion of linear geophysical data. In: International Congress of the Brazilian Geophysical Society, 3., Rio de Janeiro, Brazil, volume 1, 111-116.

CRAVEN P \& WAHBA G. 1979. Smoothing noisy data with spline functions - estimating the correct degree of smoothing by the method of generalized cross validation. Numerische Mathematik, 31: 377-403.

DEVANEY AJ. 1984. Geophysical diffraction tomography. Institute of Electrical and Electronics Engineers Transactions on Geoscience and Remote Sensing, 22: 3-13.

DICKENS TA. 1994. Diffraction tomography for crosswell imaging of nearly layered media. Geophysics, 59: 694-706.

GASSMANN F. 1951. Uber die Elastizität poröser Medien. Vierteljahrsschrift der Naturforschenden Gesellschaft in Zürich, 96, 1-23. Translated and reprinted as: On elasticity of porous media. In: PELISSIER MA (Ed.). Classics of Elastic Wave Theory, Society of Exploration Geophysicists, Tulsa (OK), 2007.

HARRIS JM. 1987. Diffraction tomography with discrete arrays of sources and receivers. Institute of Electrical and Electronics Engineers Transactions on Geoscience and Remote Sensing, 25: 448-455.

HARRIS JM \& WANG GY. 1996. Diffraction tomography for inhomogeneities in layered background medium. Geophysics, 61: 570-583.

JAYNESET. 1957. Information theory and statistical mechanics. Physical Review, 106: 620-630.

LO TW \& INDERWIESEN PL. 1994. Fundamentals of Seismic Tomography. Society of Exploration Geophysicists, Tulsa (OK), 178 pp.

MENKE W. 1989. Geophysical Data Analysis: Discrete Inverse Theory. 2nd ed., Academic Press, San Diego, 289 pp.
PENROSE R. 1955. A Generalized inverse for matrices. Proceedings of the Cambridge Philosophical Society, 51: 406-413.

ROCHA FILHO AA, HARRIS JM \& BASSREI A. 1996. A simple matrix formulation diffraction tomography algorithm. In: Brazilian Congress of Geology, 39., Salvador, Brazil, v. 2, 312-315.

SANTOS ETF \& BASSREI A. 2007. L- and Theta-curve approaches for the selection of regularization parameter in geophysical diffraction tomography. Computers \& Geosciences, 33: 618-629.

SANTOS ETF, HARRIS JM, BASSREI A \& COSTA JC. 2009. Trigonal meshes in diffraction tomography with optimum regularization: an application for carbon sequestration monitoring. Journal of Seismic Exploration, 18: 135-156.

SCHIITT H, WIGAND M \& SPANGENBERG E. 2005. Geophysical and geochemical effects of supercritical $\mathrm{CO}_{2}$ on sandstones, in Carbon Dioxide Capture for Storage in Deep Geologic Formations - Results from the $\mathrm{CO}_{2}$ Capture Project: Volume $2-$ Geologic Storage of Carbon Dioxide with Monitoring and Verification, 767-786, THOMAS DC \& BENSON SM. (Eds.). Elsevier, Amsterdam.

SHANNON CE \& WEAVER W. 1949. The Mathematical Theory of Communication. The University of Illinois Press, Urbana (IL), 144 pp.

SILVA CJMG \& BASSREI A. 2009. Regularization of inverse problems by selection of singular values: application in iterative traveltime tomography. In: International Congress of the Brazilian Geophysical Society, 11., Salvador, Brazil, 1-6.

SMITH TD, SONDERGELD CH \& RAI SC. 2003. Gassman fluid substitutions: A tutorial. Geophysics, 68: 430-440.

TIKHONOV AN \& ARSENIN VY. 1977. Solutions of III-Posed Problems. John Wiley \& Sons, Washington, 258 pp.

TWOMEY S. 1963. On the numerical solution of Fredholm integral equations of the first kind by the inversion of the linear system produced by quadrature. Journal of the Association of Computing Machines, 10 : 97-101.

WAHBA G. 1990. Spline Model for Observational Data. SIAM, Philadelphia, 169 pp.

WANG ZZ. 2001. Fundamental of rock physics. Geophysics, 66: 398412.

WU R-S \& TOKSÖZ MN. 1987. Diffraction tomography and multisource holography applied to seismic imaging. Geophysics, 52: 11-25. 